\title{
IMPACTO DO CONTROLE DA COLESTEROLEMIA: DIETA VERSUS MEDICAMENTOS
}

Nos últimos anos, foi conseguido um considerável progresso na compreensão da aterogênese e suas complicações. A aterosclerose deixou de ser vista como uma doença de depósito lipídico e passou a ser considerada uma doença inflamatória, profundamente influenciada por aspectos ambientais e genéticos.

A doença tem início na infância e parece ser muito influenciada pelo tabagismo, sedentarismo, obesidade, dieta hipercalórica com alto teor de colesterol e gordura saturada, condições que se associam a outros clássicos fatores de risco como a hipertensão arterial, diabetes e dislipidemias.

Infelizmente, a prevenção primária da doença com um custo muito menor e grande alcance epidemiológico ainda é muito negligenciada. Neste contexto, um estilo de vida mais saudável, uma dieta mais apropriada, incorporando também alguns alimentos funcionais de baixo custo (como a soja) e as fibras, poderiam reduzir a elevada incidência de complicações cardiovasculares em nosso meio.

Dispomos hoje de medicamentos muito eficazes para a obtenção de metas estabelecidas pelas recentes diretrizes das sociedades de cardiologia no Brasil e do exterior. Para pacientes de alto risco cardiovascular ou dislipidemias mais graves, as modificações de estilo de vida, mesmo incorporando uma dieta apropriada, tem se mostrado pouco eficazes tanto para que se atinjam os níveis lipídicos alvos, como para uma mudança na história natural da doença aterosclerótica que seja comparável à obtida por medicamentos como as vastatinas.

Nos dias atuais, o custo do tratamento das complicações cardiovasculares é muito elevado, principalmente na sua fase hospitalar, com procedimentos invasivos, cirurgias e medicamentos antitrombóticos de alto custo, além de internações prolongadas por complicações incapacitantes, como a insuficiência cardíaca grave ou acidente vascular cerebral.

Algumas análises de farmacoeconomia têm mostrado a viabilidade do tratamento das dislipidemias, sobretudo para pacientes de alto risco, desde que se atinjam as metas propostas. Modificações mais discretas do perfil lipídico e o uso irregular de medica- mentos não reduzem os eventos cardiovasculares e apenas acrescentam mais um ônus econômico.

Embora uma dieta apropriada deva ser sempre recomendada, sua completa assimilação pelos nossos pacientes ainda é modesta, e ainda existem controvérsias sobre o valor de alguns de seus componentes. Embora seu valor estratégico seja muito relevante para a redução dos eventos cardiovasculares, sobretudo na prevenção primária da doença, as modificações de alguns parâmetros de estabilidade da doença (atividade inflamatória, hemostasia, vasorreatividade etc.), não são atingidas em curto prazo.

Quanto aos fármacos hipolipemiantes, estes são custo-efetivos desde que utilizados de maneira apropriada, de acordo com as diretrizes atuais, especialmente para os pacientes de maior risco cardiovascular. Com base em recentes estudos, estes fármacos parecem atenuar significativamente o impacto dos fatores de risco, como a hipertensão arterial e $\circ$ diabetes, mesmo em indivíduos com níveis lipídicos apenas discretamente alterados. 